

\title{
On a continuum thermodynamics formulation and computational aspects of finite growth in soft tissues \\ Boumediene Nedjar
}

\section{To cite this version:}

Boumediene Nedjar. On a continuum thermodynamics formulation and computational aspects of finite growth in soft tissues. International Journal for Numerical Methods in Biomedical Engineering, 2011, 27, pp.1850-1866. 10.1002/cnm.1448 . hal-00668026

\section{HAL Id: hal-00668026 \\ https: / hal-enpc.archives-ouvertes.fr/hal-00668026}

Submitted on 8 Feb 2012

HAL is a multi-disciplinary open access archive for the deposit and dissemination of scientific research documents, whether they are published or not. The documents may come from teaching and research institutions in France or abroad, or from public or private research centers.
L'archive ouverte pluridisciplinaire HAL, est destinée au dépôt et à la diffusion de documents scientifiques de niveau recherche, publiés ou non, émanant des établissements d'enseignement et de recherche français ou étrangers, des laboratoires publics ou privés. 


\title{
On a continuum thermodynamics formulation and computational aspects of finite growth in soft tissues
}

\author{
B. Nedjar* \\ Université Paris-Est, Laboratoire Navier (ENPC/IFSTTAR/CNRS), Ecole des Ponts ParisTech, 6 et 8 avenue Blaise \\ Pascal, 77455 Marne-la-Vallée, France
}

\begin{abstract}
SUMMARY
In this paper, we try to settle the bases of a concise modelling of growth within the unified framework of continuum thermodynamics. Special emphasis is placed on the modelling of soft biological tissues at finite strains. For this, we adopt the nowadays well known kinematic assumption of a multiplicative decomposition of the deformation gradient into an elastic part and a growth part. It is shown how continuum thermodynamics is crucial in setting convenient forms for the coupling between stress and growth in general. The particularization to isotropy simplifies considerably the growth modelling from both the theoretical and the numerical points of view. Simple growth constitutive equations are proposed and embedded into a finite element context. Finally, representative numerical examples examining stress-dependent growth and residual stress arising from growth and resorption close this study. Copyright (c) 2010 John Wiley \& Sons, Ltd.
\end{abstract}

Received...

KEY WORDS: soft biological tissue; growth; continuum thermodynamics; finite element method

\section{INTRODUCTION}

During recent years, much interest has been devoted to the modelling of biological tissues, a challenging task in the field of computational biomechanics. Living tissues continuously change and adapt where growth and remodelling are fundamental mechanical processes in their normal development and in various pathological conditions. On one hand, growth is primarily thought as a volume change with the mass change following from it. And on the other hand, remodelling (change in properties) can also lead to mass change if the structural changes are related to the density changes. The reader is referred for example to $(1 ; 2 ; 3 ; 4 ; 5)$ for extensive discussions concerning these topics.

The present paper deals with biomechanical aspects of growth stimulated by mechanical quantities such as the stress and/or the strain in the tissue. We focus here on a formulation for finite growth and finite deformation. More precisely, and motivated by the continuum theory proposed by

$\overline{{ }^{*} \text { Correspondence }}$ to: boumediene.nedjar@enpc.fr, Tel. +33-1-64153715

Copyright (C) 2010 John Wiley \& Sons, Ltd.

Prepared using cnmauth.cls [Version: 2010/03/27 v2.00] 
Rodriguez et al. (1994) in (6), the key kinematical assumption is a multiplicative decomposition of the deformation gradient into a growth part and an elastic (reversible) part, see also $(2 ; 7 ; 8 ; 9)$ among others. Moreover, density is considered here to be constant in time and position, an assumption also adopted in earlier works, for instance see $(10 ; 11)$ among others. The growth consists then of the additional or removal of the same tissue material.

Embedded into the classical continuum thermodynamics framework, these considerations lead to remarkeable properties and match ideas used in earlier works, e.g. the references cited above for example. Growth being considered here as a dissipative process, it is shown how the effect of growth on stress and the effect of stress on growth become evident. The constitutive relations and the characterization of the growth evolution are then constructed in accordance with the continuum thermodynamics requirements. From this framework, it naturally appears that stress is the biomechanical factor which drives growth. This agrees with many recent contributions in the modelling of soft tissues, see for example $(12 ; 13)$ for the growth modelling in arteries. Other theories are based on strain-induced or strain-energy driving growth. However, and as mentioned for instance in (2), in the absence of a universal mechano-growth law, experimental data can be found to support each of these mechanical quantities to modulate the growth.

When particularized to isotropy, the above general continuum formulation simplifies considerably. On the theoretical side, it is deduced that the growth evolution relates the stress tensor to the symmetric growth-rate tensor. This later quantity was first defined in (6). Simple growth laws can be proposed and/or adapted from previous ideas developed in the literature. For instance, growth or resorption can occur so that the stress due to loading returns to an equilibrium state. And on the numerical side, the whole procedure is very similar to the current state of the art in the finite element treatment of elastoplasticity. In particular, use is made of the so called exponential map to approximate the (local) growth evolution equations. This procedure is detailed for each of the model examples proposed in this work.

An outline of the remainder of the paper is as follows. The adopted kinematic assumption is recalled in Section 2. Then, the finite strain growth formulation is developed in Section 3 where the general form of stress-growth couplings is established in accordance with continuum thermodynamics. In Section 4, the particularization to isotropy is developed from both the theoretical and the numerical points of view. A special attention is devoted to the algorithmic update of the local growth evolution equations. The efficiency of the proposed framework is then evaluated numerically through a set of representative numerical examples in Section 5. And finally, conclusions are drawn in the last section. Interesting properties will be highlighted throughout the mathematical developments in this paper with noteworthy remarks and comments.

\section{OVERVIEW OF THE KINEMATIC ASSUMPTIONS}

As a point of departure, we adopt the nowadays well accepted kinematic assumption of a multiplicative decomposition of the overall deformation gradient $\boldsymbol{F}$ into an elastic part $\boldsymbol{F}^{\mathrm{e}}$ and a (stress-free) growth part $\boldsymbol{F}^{\mathrm{g}}$, as introduced by Rodriguez et al. in (6):

$$
\boldsymbol{F}=\boldsymbol{F}^{\mathrm{e}} \boldsymbol{F}^{\mathrm{g}} \text {. }
$$


Here $\boldsymbol{F}^{\mathrm{e}}$ is the part of the deformation gradient comprising the deformation gradient giving rise to residual elastic stress $\boldsymbol{F}^{\mathrm{r}}$ and the deformation gradient due to external loading $\boldsymbol{F}^{\ell}$, see e.g. (2). Hence we also have the sub-decomposition $\boldsymbol{F}^{\mathrm{e}}=\boldsymbol{F}^{\ell} \boldsymbol{F}^{\mathrm{r}}$, see the sketch in Figure 1 for an illustration. For instance, and in the absence of external loads $\left(\boldsymbol{F}^{\mathrm{e}} \equiv \boldsymbol{F}^{\mathrm{r}}\right)$, the part $\boldsymbol{F}^{\mathrm{r}}$ can be computed as an elastic deformation that ensures the continuity of the body. In fact, there is no requirement that the growth part $\boldsymbol{F}^{\mathrm{g}}$ corresponds to a compatible displacement field, i.e. for example, a cell may grow independently of its neighbors. However, if residual stress is not of interest, it is not necessary to compute $\boldsymbol{F}^{\mathrm{r}}$. And in all cases, $\boldsymbol{F}^{\mathrm{e}}$ can be used as the principal deformation variable.

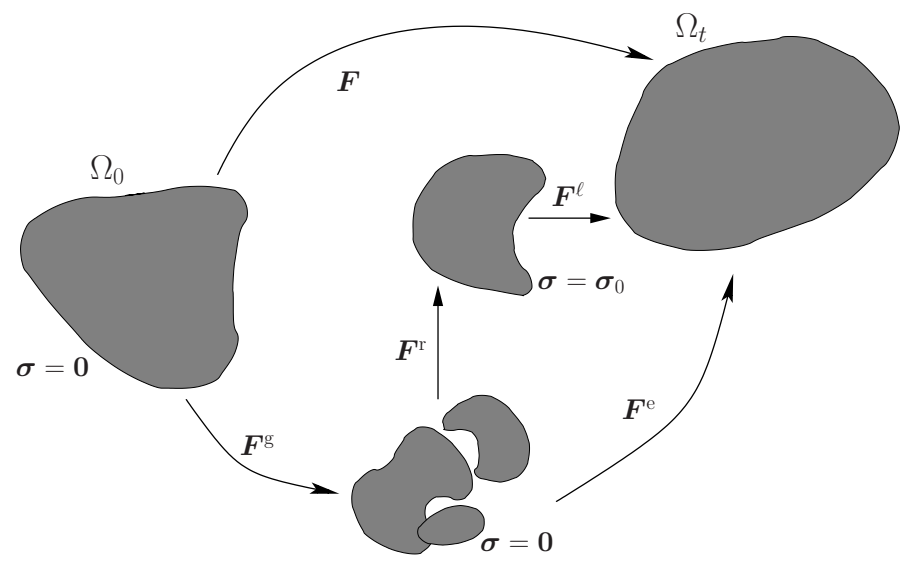

Figure 1. Local decomposition of the deformation gradient for finite growth (after (2) modified from (6)).

Last but not least, notice that since growth is not an isochoric process, we have $J^{\mathrm{g}}=\operatorname{det}\left[\boldsymbol{F}^{\mathrm{g}}\right] \neq 1$ for the growth Jacobian. One has then $J^{\mathrm{g}}>1$ for growth (added volume), and $J^{\mathrm{g}}<1$ for resorption (removed volume).

\section{CONSTITUTIVE MODELLING OF GROWTH}

The next step is to account for the effects of growth on stress together with the effects of stress on growth. It is shown here that the continuum thermodynamics framework constitutes a useful tool to naturally exhibit those mutual phenomena.

In general, the elastic behaviour is assumed to be characterized by a free energy function $\psi$ of the form

$$
\psi=\psi\left(\boldsymbol{C}^{\mathrm{e}}\right),
$$

where $C^{\mathrm{e}}=\boldsymbol{F}^{\mathrm{e}^{T}} \boldsymbol{F}^{\mathrm{e}}$ is the elastic right Cauchy-Green tensor. Here and in all what follows, the notation $(.)^{T}$ is used for the transpose operator. The dependence of $\psi$ on $\boldsymbol{F}^{\mathrm{e}}$ through $\boldsymbol{C}^{\mathrm{e}}$ follows from the fundamental principle of material frame indifference, e.g. see (14).

By its nature, growth is considered as a dissipative phenomenon. Then, confining our attention to isothermal processes, the second law of continuum thermodynamics is given by the following 
expression in the form of the Clausius-Duhem dissipation inequality:

$$
\mathcal{D}=\boldsymbol{S}: \frac{1}{2} \dot{\boldsymbol{C}}+\dot{\varsigma}-\dot{\psi} \geq 0
$$

where $\boldsymbol{S}$ is the (symmetric) second Piola-Kirchhoff stress tensor, and $\boldsymbol{C}=\boldsymbol{F}^{T} \boldsymbol{F}$ denotes the (total) right Cauchy-Green tensor. In eqn. (3), $\dot{\zeta}$ is a nonmechanical and nonthermal energy supply source of an electrochemical and/or biological nature assumed to be present in living materials such as soft tissues. For instance, a similar quantity has also been introduced in the fibre reorientation remodelling theory proposed in (15). For notational purposes it is written in rate form, but it is not necessarily the time derivation of a scalar function. Its dimension is a volumetric energy per unit of time.

Inserting the general expression (2) into (3), and use of the kinematic relation $\boldsymbol{C}=\boldsymbol{F}^{\mathrm{g}^{T}} \boldsymbol{C}^{\mathrm{e}} \boldsymbol{F}^{\mathrm{g}}$, give after simple algebraic manipulations exploiting tensors' symmetries

$$
\mathcal{D}=\left[\overline{\boldsymbol{S}}-2 \frac{\partial \psi}{\partial \boldsymbol{C}^{\mathrm{e}}}\right]: \frac{1}{2} \dot{\boldsymbol{C}}^{\mathrm{e}}+\dot{\boldsymbol{\zeta}}+\boldsymbol{C}^{\mathrm{e}} \overline{\boldsymbol{S}}: \boldsymbol{l}^{\mathrm{g}} \geq 0
$$

where $\overline{\boldsymbol{S}}=\boldsymbol{F}^{\mathrm{g}} \boldsymbol{S} \boldsymbol{F}^{\mathrm{g}^{T}}$ is the second Piola-Kirchhoff stress tensor in the (local) intermediate configuration defined by $\boldsymbol{F}^{\mathrm{g}}$, and where $\boldsymbol{l}^{\mathrm{g}}=\dot{\boldsymbol{F}}^{\mathrm{g}} \boldsymbol{F}^{\mathrm{g}}{ }^{-1}$ is the left-rate tensor of growth distorsion. This latter is the work-conjugate of the Mandel-like stress tensor $\boldsymbol{C}^{\mathrm{e}} \overline{\boldsymbol{S}}$.

Remark 1. Notice that the last term in the left hand side of (4) can be equivalently written through the following stress-power relationship:

$$
\boldsymbol{C}^{\mathrm{e}} \overline{\boldsymbol{S}}: \boldsymbol{l}^{\mathrm{g}}=\boldsymbol{C S}: \boldsymbol{L}^{\mathrm{g}}
$$

where, this time, $\boldsymbol{L}^{\mathrm{g}}=\boldsymbol{F}^{\mathrm{g}^{-1}} \dot{\boldsymbol{F}}^{\mathrm{g}}$ is the right-rate tensor of growth distorsion. It is the work-conjugate of the material stress tensor $\boldsymbol{C S}$. This latter is called an Eshelby-like stress tensor in (16) as, up to a spherical term and a sign, it coincides with it. However, one can also say it is a Mandel-like stresstensor, the Mandel stress being defined with respect to the so-called intermediate configuration.

\subsection{Effects of growth on stress.}

By standard arguments of continuum thermodynamics, see for example $(17 ; 18)$, we end up with the following expression for the stress tensor:

$$
\overline{\boldsymbol{S}}=2 \frac{\partial \psi}{\partial \boldsymbol{C}^{\mathrm{e}}}
$$

This general constitutive relation clearly traduces the effects of growth on stress since the elastic right Cauchy-Green tensor $C^{\mathrm{e}}$ depends on the growth deformation variable $\boldsymbol{F}^{\mathrm{g}}$, i.e. $C^{\mathrm{e}}=$ $\boldsymbol{F}^{\mathrm{g}^{-T}} \boldsymbol{C} \boldsymbol{F}^{\mathrm{g}^{-1}}$. 


\subsection{Effects of stress on growth.}

We continue now with the description of the induced growth in the soft tissue. Taking into account the constitutive relation (6) into (4), the reduced dissipation becomes

$$
\mathcal{D}=\dot{\zeta}+\boldsymbol{\Sigma}: \boldsymbol{l}^{\mathrm{g}} \geq 0
$$

where we have introduced the notation $\boldsymbol{\Sigma}=\boldsymbol{C}^{\mathrm{e}} \overline{\boldsymbol{S}}$ for the generally nonsymmetric Mandel-like stress tensor.

Then, one has to define an evolution equation for the rate quantity $l^{g}$. In accordance with the expression (7), a natural choice is to assume this rate to depend on its work-conjugate thermodynamic force $\boldsymbol{\Sigma}$. Such a relation may be either of a simple or a complicated form. At this stage of the study, we consider a general expression written as

$$
l^{\mathrm{g}}=\Gamma(\boldsymbol{\Sigma}),
$$

where $\boldsymbol{\Gamma}(\boldsymbol{\Sigma})$ is a tensor valued function. Furthermore, using the definition of the left-rate $\boldsymbol{l}^{\mathrm{g}}$, the relation (8) can be equivalently written as

$$
\dot{\boldsymbol{F}}^{\mathrm{g}}=\boldsymbol{\Gamma}(\boldsymbol{\Sigma}) \boldsymbol{F}^{\mathrm{g}}
$$

where one can clearly notice the effect of stress on growth through the tensorial function $\boldsymbol{\Gamma}(\boldsymbol{\Sigma})$. This general expression describes the direct evolution of the nonsymmetric growth deformation gradient. And from the numerical point of view, its approximation in the solution process could be non trivial. However, algorithms for the evaluation of the exponential map of a nonsymmetric argument have been developed for similar local evolution equations in finite viscoplasticity, see for example $(19 ; 20)$. The isotropic case discussed below simplifies considerably this task.

Remark 2. With the general evolution equation (8), the scalar valued product $\Sigma: \Gamma(\boldsymbol{\Sigma})$ could be positive or negative, unless $\boldsymbol{\Gamma}(\boldsymbol{\Sigma})$ is derived from a (convex) pseudo-potential function $\phi(\boldsymbol{\Sigma})$, i.e. $\boldsymbol{\Gamma}(\boldsymbol{\Sigma})=\partial_{\boldsymbol{\Sigma}} \phi(\boldsymbol{\Sigma})$, in which case it would be always positive. Hence, in general, an energy supply in the form of nonzero values for the source term $\dot{\zeta}$ is needed in order to always satisfy the dissipation inequality (7).

\section{ISOTROPIC FINITE GROWTH}

Of interest for the developments presented below is the consideration of the isotropic case. In our context, this restriction is assumed on the intermediate configuration defined by $\boldsymbol{F}^{\mathrm{g}}$. The free energy function $\psi$ in eqn. (2) equivalently depends on the elastic left Cauchy-Green tensor $\boldsymbol{b}^{\mathrm{e}}=\boldsymbol{F}^{\mathrm{e}} \boldsymbol{F}^{\mathrm{e}^{T}}$, i.e. $\psi=\psi\left(\boldsymbol{b}^{\mathrm{e}}\right)$. Then, and as a consequence, the constitutive relation (6) can equivalently be written as, see e.g. $(14 ; 21)$,

$$
\boldsymbol{\tau}=2 \frac{\partial \psi}{\partial \boldsymbol{b}^{\mathrm{e}}} \boldsymbol{b}^{\mathrm{e}},
$$

where $\boldsymbol{\tau}=J \boldsymbol{\sigma}$ ( $\equiv \boldsymbol{F} \boldsymbol{S} \boldsymbol{F}^{T}$ ) is the (symmetric) Kirchhoff stress tensor, $\boldsymbol{\sigma}$ being the true Cauchy stress tensor and $J=\operatorname{det}[\boldsymbol{F}]>0$ is the Jacobian of the overall deformation. 
Combining (10) with the relation $\overline{\boldsymbol{S}}=\boldsymbol{F}^{\mathrm{e}^{-1}} \boldsymbol{\tau} \boldsymbol{F}^{\mathrm{e}^{-T}}$, replacing the result into (7), and exploiting again the tensors' symmetries, lead to:

$$
\mathcal{D}=\dot{\varsigma}+2 \frac{\partial \psi}{\partial \boldsymbol{b}^{\mathrm{e}}}: \boldsymbol{F}^{\mathrm{e}} \boldsymbol{d}^{\mathrm{g}} \boldsymbol{F}^{\mathrm{e}^{T}} \geq 0
$$

where

$$
\boldsymbol{d}^{\mathrm{g}}=\operatorname{sym}\left[\boldsymbol{l}^{\mathrm{g}}\right]=\frac{1}{2}\left(\dot{\boldsymbol{F}}^{\mathrm{g}} \boldsymbol{F}^{\mathrm{g}^{-1}}+\boldsymbol{F}^{\mathrm{g}^{-T}} \dot{\boldsymbol{F}}^{\mathrm{g}^{T}}\right)
$$

is no more than the growth rate-of-deformation tensor as first defined in (6), see also (2).

Expression (11) can again be rearranged. In fact, and on one hand, exploiting the kinematic relation $\boldsymbol{b}^{\mathrm{e}}=\boldsymbol{F} \boldsymbol{C}^{\mathrm{g}^{-1}} \boldsymbol{F}^{T}$, where $\boldsymbol{C}^{\mathrm{g}}=\boldsymbol{F}^{\mathrm{g}^{T}} \boldsymbol{F}^{\mathrm{g}}$ is the growth right Cauchy-Green tensor, one can define the Lie derivative of $\boldsymbol{b}^{\mathrm{e}}$ as, see e.g. (22),

$$
\mathcal{L}_{v} b^{\mathrm{e}}=\boldsymbol{F} \frac{\mathrm{d}}{\mathrm{d} t}\left[\boldsymbol{C}^{\mathrm{g}^{-1}}\right] \boldsymbol{F}^{T}
$$

An easy calculation then gives the identity

$$
-\frac{1}{2} \mathcal{L}_{v} \boldsymbol{b}^{\mathrm{e}}=\boldsymbol{F}^{\mathrm{e}} \boldsymbol{d}^{\mathrm{g}} \boldsymbol{F}^{\mathrm{e}^{T}}
$$

And on the other hand, replacing this last result into (11) and using the constitutive relation (10) lead then to the following reduced dissipation inequality relative to the isotropic case:

$$
\mathcal{D}=\dot{\zeta}+\boldsymbol{\tau}:\left[-\frac{1}{2}\left(\mathcal{L}_{v} \boldsymbol{b}^{\mathrm{e}}\right) \boldsymbol{b}^{\mathrm{e}^{-1}}\right] \geq 0
$$

which must hold for all admissible process in the soft biological tissue.

Now for the description of the (local) growth evolution, one has to define a relation for the rate quantity $-\frac{1}{2}\left(\mathcal{L}_{v} \boldsymbol{b}^{\mathrm{e}}\right) \boldsymbol{b}^{\mathrm{e}^{-1}}$. In view of the expression (15), a natural choice is to assume this later to depend on the Kirchhoff stress tensor $\tau$. We consider again a general form written this time as

$$
-\frac{1}{2}\left(\mathcal{L}_{v} \boldsymbol{b}^{\mathrm{e}}\right) \boldsymbol{b}^{\mathrm{e}^{-1}}=\boldsymbol{g}(\boldsymbol{\tau}) \quad \Rightarrow \quad \mathcal{L}_{v} \boldsymbol{b}^{\mathrm{e}}=-2 \boldsymbol{g}(\boldsymbol{\tau}) \boldsymbol{b}^{\mathrm{e}}
$$

where $\boldsymbol{g}(\boldsymbol{\tau})$ is a tensor valued function which, besides on its lonely argument $\boldsymbol{\tau}$, can also depend on other variables, but these latters can only act as parameters, i.e. $\boldsymbol{g} \equiv \boldsymbol{g}(\boldsymbol{\tau} ; \ldots)$. Notice that from (16), the growth response reduces to the evolution of the (symmetric) spatial tensor $\boldsymbol{b}^{\mathrm{e}}$ defining the stress through the constitutive relation (10).

Remark 3. Notice that the tensorial function $\boldsymbol{g}(\boldsymbol{\tau})$ is restricted by material frame indifference to isotropic functions. That is,

$$
\boldsymbol{g}\left(\boldsymbol{Q} \boldsymbol{\tau} \boldsymbol{Q}^{T}\right)=\boldsymbol{g}(\boldsymbol{\tau}), \quad \forall \boldsymbol{Q} \in S O(3),
$$

where $S O(3)$ is the finite group of rotations. 
Remark 4. Use of the definition (13) together with the kinematic relation $\boldsymbol{C}^{\mathrm{g}^{-1}}=\boldsymbol{F}^{-1} \boldsymbol{b}^{\mathrm{e}} \boldsymbol{F}^{-T}$ into the evolution equation $(16)_{2}$, we get

$$
\frac{\mathrm{d}}{\mathrm{d} t}\left[\boldsymbol{C}^{\mathrm{g}^{-1}}\right]=-2 \widetilde{\boldsymbol{G}} \boldsymbol{C}^{\mathrm{g}^{-1}} \text { for } \quad \widetilde{\boldsymbol{G}}=\boldsymbol{F}^{-1} \boldsymbol{g} \boldsymbol{F}
$$

This last expression allows the integration of the same growth evolution equation, but this time in the material reference configuration.

Remark 5. Comparing the evolution equations (8) and (16), and using the kinematic definitions (12) and (14), the tensorial functions $\boldsymbol{g}$ and $\boldsymbol{\Gamma}$ are related as

$$
\boldsymbol{g}(\boldsymbol{\tau})=\boldsymbol{F}^{\mathrm{e}} \operatorname{sym}\left[\boldsymbol{\Gamma}\left(\boldsymbol{\Sigma}=\boldsymbol{F}^{\mathrm{e}^{T}} \boldsymbol{\tau} \boldsymbol{F}^{\mathrm{e}^{-T}}\right)\right] \boldsymbol{F}^{\mathrm{e}^{-1}}
$$

To get further insight into the nature of the present general form of growth response, the tensorial equation $(16)_{2}$ implies the following evolution for the rate of growth volume change:

$$
\frac{\mathrm{d}}{\mathrm{d} t}\left[\ln \left[J^{\mathrm{g}}\right]\right]=\operatorname{tr}[\boldsymbol{g}(\boldsymbol{\tau})] .
$$

where we recall that $J^{\mathrm{g}}=\operatorname{det}\left[\boldsymbol{F}^{\mathrm{g}}\right]$ is the growth volume change.

To prove this result, let us first recall that time differentiation of $\boldsymbol{b}^{\mathrm{e}}=\boldsymbol{F} \boldsymbol{C}^{\mathrm{g}^{-1}} \boldsymbol{F}^{T}$ yields the identity

$$
\dot{b}^{\mathrm{e}}=\boldsymbol{l} \boldsymbol{b}^{\mathrm{e}}+\boldsymbol{b}^{\mathrm{e}} \boldsymbol{l}^{T}+\mathcal{L}_{v} \boldsymbol{b}^{\mathrm{e}}
$$

where $\boldsymbol{l}=\dot{\boldsymbol{F}} \boldsymbol{F}^{-1}$ is the spatial velocity gradient. The elastic volume change being given by $J^{\mathrm{e}}=\operatorname{det}\left[\boldsymbol{F}^{\mathrm{e}}\right]=\left(\operatorname{det}\left[\boldsymbol{b}^{\mathrm{e}}\right]\right)^{1 / 2}>0$, the proof follows from (21) along with (16), since

$$
\dot{J}^{\mathrm{e}}=\frac{1}{2} J^{\mathrm{e}} \boldsymbol{b}^{\mathrm{e}^{-1}}: \dot{b}^{\mathrm{e}}=J^{\mathrm{e}}\left[\operatorname{tr}[\boldsymbol{d}]+\frac{1}{2} \mathcal{L}_{v} \boldsymbol{b}^{\mathrm{e}}: \boldsymbol{b}^{\mathrm{e}^{-1}}\right]=J^{\mathrm{e}} \operatorname{tr}[\boldsymbol{d}-\boldsymbol{g}(\boldsymbol{\tau})]
$$

where $\boldsymbol{d}=\operatorname{sym}[\boldsymbol{l}]$ denotes the rate of (total) deformation gradient tensor. Inserting the relations $J=J^{\mathrm{e}} J^{\mathrm{g}}$ and $\dot{J}=J \operatorname{tr}[\boldsymbol{d}]$ into (22) gives (20).

Moreover, using the identity $\dot{J}^{\mathrm{g}}=J^{\mathrm{g}} \operatorname{tr}\left[\boldsymbol{d}^{\mathrm{g}}\right]$, the evolution equation (20) can equivalently be written as

$$
\frac{1}{V} \frac{\mathrm{d} V}{\mathrm{~d} t} \equiv \operatorname{tr}\left[\boldsymbol{d}^{\mathrm{g}}\right]=\operatorname{tr}[\boldsymbol{g}(\boldsymbol{\tau})]
$$

where $V$ is the growth volume as defined in (23), see also (2).

\subsection{A growth modelling example}

The modelling framework developed above is independent of the particular choices made for the elastic behaviour and the growth evolution in soft tissues. However, and in the light of the lack of appropriate experiments, we consider for simplicity that the growth process is uniform in all directions. That is, the tensorial function $\boldsymbol{g}(\boldsymbol{\tau})$ simplifies to a spherical form as

$$
\boldsymbol{g}(\boldsymbol{\tau})=g(\boldsymbol{\tau}) \boldsymbol{I}
$$


where $g(\boldsymbol{\tau})$ is here a scalar valued isotropic function, and where $\boldsymbol{I}$ is the second order identity tensor. With this specific type of evolution law, the rate of volumetric growth becomes, see eqn. (23),

$$
\operatorname{tr}\left[\boldsymbol{d}^{\mathrm{g}}\right]=3 g(\boldsymbol{\tau})
$$

Examples for the general function $g(\boldsymbol{\tau} ; \ldots)$ will be precised in the applications' section below.

\subsection{Integration algorithm}

We formulate in this section a numerical algorithm to integrate the constitutive relations developed above. The algorithmic approximation mimics the principal steps adopted in modern finite element treatments of elastoplasticity and viscoelasticity at finite strains based on multiplicative decomposition kinematics.

In the isotropic case, and at a given material point, we consider known the solution at time $t_{n}$, consisting of the deformation gradient $\boldsymbol{F}_{n}$ and the internal variable, say $\boldsymbol{C}_{n}^{\mathrm{g}^{-1}}$ (or $\boldsymbol{b}_{n}^{\mathrm{e}}$ since $\boldsymbol{b}_{n}^{\mathrm{e}}=\boldsymbol{F}_{n} \boldsymbol{C}_{n}^{\mathrm{g}^{-1}} \boldsymbol{F}_{n}^{T}$ ). Given a deformation gradient $\boldsymbol{F}_{n+1}$ at $t_{n+1}$, we are interested in the stresses and the updated internal variable $C_{n+1}^{\mathrm{g}^{-1}}$.

An appropriate approximation of the general evolution equation $(16)_{2}$ is given by the exponential map

$$
\boldsymbol{b}_{n+1}^{\mathrm{e}}=\exp \left[-2 \Delta t g_{n+1}\right] \boldsymbol{b}_{n+1}^{\mathrm{etr}},
$$

where $\Delta t=t_{n+1}-t_{n}$, and $\boldsymbol{b}_{n+1}^{\mathrm{etr}}$ is the trial state defined from the initial conditions and the application of the operator split to $(16)_{2}$, see for example $(24 ; 25)$ and references therein for similarities with the elastoplastic case,

$$
\boldsymbol{b}_{n+1}^{\mathrm{etr}}=\boldsymbol{F}_{n+1} \boldsymbol{C}_{n}^{\mathrm{g}^{-1}} \boldsymbol{F}_{n+1}^{T} \text {. }
$$

In deriving (26), use has been made of the particularization (24) where the growth scalar function is evaluated at $\boldsymbol{\tau}_{n+1}$, i.e. $g_{n+1}=g\left(\boldsymbol{\tau}_{n+1} ; \ldots\right)$, and where in turn, $\boldsymbol{\tau}_{n+1}$ is defined by the constitutive relation (10) evaluated at $\boldsymbol{b}_{n+1}^{\mathrm{e}}$. The discrete equation (26) simplifies considerably when written in the principal frame. In fact, on one hand, let $\boldsymbol{n}_{n+1}^{(A)}$ and $\left(\lambda_{A n+1}^{\mathrm{e}}\right)^{2}, A=1,2,3$, denote the principal directions and principal values of $\boldsymbol{b}_{n+1}^{\mathrm{e}}$. And on the other hand, let $\boldsymbol{n}_{n+1}^{(A) \operatorname{tr}}$ and $\left(\lambda_{A n+1}^{\mathrm{e} t \mathrm{tr}}\right)^{2}, A=1,2,3$, be the corresponding quantities for $\boldsymbol{b}_{n+1}^{\mathrm{e} t \mathrm{r}}$. Then, from the uniqueness of the spectral decomposition, (26) implies $\boldsymbol{n}_{n+1}^{(A)}=\boldsymbol{n}_{n+1}^{(A) \text { tr }}, A=1,2,3$, for the principal directions and

$$
\left(\lambda_{A n+1}^{\mathrm{e}}\right)^{2}=\exp \left[-2 \Delta t g_{n+1}\right]\left(\lambda_{A n+1}^{\mathrm{etr}}\right)^{2}, \quad A=1,2,3 .
$$

Moreover, taking the natural logarithm of this later expression, we obtain

$$
\varepsilon_{A n+1}^{\mathrm{e}}=\varepsilon_{A n+1}^{\mathrm{etr}}-\Delta g_{n+1}, \quad A=1,2,3
$$

for the logarithmic elastic principal stretch $\varepsilon_{A n+1}^{\mathrm{e}}=\ln \left[\lambda_{A n+1}^{\mathrm{e}}\right]$ and its trial counterpart.

The isotropy assumption also implies that the free energy $\psi$ is a function of the principal elastic streches, and therefore, a function of the principal logarithmic streches: $\psi\left(\boldsymbol{b}^{\mathrm{e}}\right)=\hat{\psi}\left(\varepsilon^{\mathrm{e}}\right)$ for 
Table I. Exponential map for isotropic finite growth.

Given $\left\{\boldsymbol{F}_{n}, \boldsymbol{b}_{n}^{\mathrm{e}}\right\}$ and $\boldsymbol{F}_{n+1}$ for a time increment $\Delta t$, then

1. Compute the initial state for the exponential map

$$
\begin{aligned}
& \quad \boldsymbol{b}_{n+1}^{\mathrm{etr}}=\boldsymbol{F}_{n+1} \boldsymbol{C}_{n}^{\mathrm{g}^{-1}} \boldsymbol{F}_{n+1}^{T} \\
& \text { for } \boldsymbol{C}_{n}^{\mathrm{g}^{-1}}=\boldsymbol{F}_{n}^{-1} \boldsymbol{b}_{n}^{e} \boldsymbol{F}_{n}^{-T} .
\end{aligned}
$$

2. Compute the spectral decomposition

$$
\boldsymbol{b}_{n+1}^{\mathrm{etr}}=\sum_{A=1}^{3}\left(\lambda_{A n+1}^{\mathrm{e} t \mathrm{r}}\right)^{2} \boldsymbol{n}_{n+1}^{(A) \operatorname{tr}} \otimes \boldsymbol{n}_{n+1}^{(A) \operatorname{tr}}
$$

and the logarithmic stretches $\varepsilon_{A n+1}^{\mathrm{etr}}=\ln \left[\lambda_{A n+1}^{\mathrm{etr}}\right]$.

3. Solve for the updated logarithmic stretches $\varepsilon_{A n+1}^{\mathrm{e}}$, eqn. (29).

4. Recover the updated tensors

$$
\begin{aligned}
\boldsymbol{b}_{n+1}^{\mathrm{e}} & =\sum_{A=1}^{3} \exp \left[2 \varepsilon_{A n+1}^{\mathrm{e}}\right] \boldsymbol{n}_{n+1}^{(A)} \otimes \boldsymbol{n}_{n+1}^{(A)} \\
\boldsymbol{\tau}_{n+1} & =\sum_{A=1}^{3} \tau_{A n+1} \boldsymbol{n}_{n+1}^{(A)} \otimes \boldsymbol{n}_{n+1}^{(A)}
\end{aligned}
$$

where $\boldsymbol{n}_{n+1}^{(A)}=\boldsymbol{n}_{n+1}^{(A) \operatorname{tr}}$ and $\tau_{A n+1}=\frac{\partial \hat{\psi}}{\partial \varepsilon_{A}^{\mathrm{e}}}\left(\varepsilon_{n+1}^{\mathrm{e}}\right)$, $A=1,2,3$.

5. Compute the algorithmic $3 \times 3$ matrix $\boldsymbol{a}_{n+1}^{\mathrm{g}}$ and the spatial tagent moduli $\widetilde{\mathbf{C}}_{n+1}$.

$\varepsilon^{\mathrm{e}}=\left[\varepsilon_{1}^{\mathrm{e}}, \varepsilon_{2}^{\mathrm{e}}, \varepsilon_{3}^{\mathrm{e}}\right]^{T}$. Moreover, the stress tensor $\boldsymbol{\tau}_{n+1}$ being coaxial with $\boldsymbol{b}_{n+1}^{\mathrm{e}}$, they share the same principal directions $\left\{\boldsymbol{n}_{n+1}^{(A)}\right\}_{A=1,2,3}$ for the principal kirchhoff stresses $\tau_{A n+1}$ given as

$$
\hat{\tau}_{n+1}=\frac{\partial \hat{\psi}}{\partial \varepsilon^{\mathrm{e}}}\left(\varepsilon_{n+1}^{\mathrm{e}}\right)
$$

where $\hat{\boldsymbol{\tau}}_{n+1}=\left[\tau_{1 n+1}, \tau_{2 n+1}, \tau_{3 n+1}\right]^{T}$. Table I summarizes the conceptual steps involved during the algorithmic treatment of the isotropic finite growth. Being growth model specific, steps 3 and 5 of Table I will be detailed in the next section for each particular model example.

4.2.1. Algorithmic tangent moduli. The initial boundary value problem of finite growth is nonlinear. The sources of nonlinearities are geometric (arising from the multiplicative kinematics) and material (arising from the nonlinearity of the constitutive relations). Hence, this problem is solved by means of an iterative process of the Newton's type. Accordingly, this requires the linearization of the global equilibrium problem about a known state at time $t_{n}$. This procedure is nowadays standard. We give here the contribution to the material part of the tangent stiffness (step 5 of Table I) where it is of interest to determine the relation between the rate of strain and the rate of stress. In spatial 
description, this takes the form

$$
\mathcal{L}_{v} \boldsymbol{\tau}_{n+1} \equiv \boldsymbol{F}_{n+1} \dot{\boldsymbol{S}}_{n+1} \boldsymbol{F}_{n+1}^{T}=\widetilde{\mathbf{C}}_{n+1}: \boldsymbol{d}_{n+1}
$$

where $\mathcal{L}_{v} \boldsymbol{\tau}_{n+1}$ is here the Lie derivatve of the Kirchhoff stress tensor, and $\widetilde{\mathbf{C}}_{n+1}$ is the fourth order spatial tangent moduli given by, see for instance $(24 ; 26)$ for details,

$$
\widetilde{\mathbf{C}}_{n+1}=\sum_{A=1,3} \sum_{B=1,3} a_{A B n+1}^{\mathrm{g}} \boldsymbol{m}_{n+1}^{(A) \operatorname{tr}} \otimes \boldsymbol{m}_{n+1}^{(A) \operatorname{tr}}+\boldsymbol{k}_{n+1}^{\mathrm{tr}}
$$

where $\boldsymbol{m}_{n+1}^{(A) \operatorname{tr}}=\boldsymbol{n}_{n+1}^{(A) \operatorname{tr}} \otimes \boldsymbol{n}_{n+1}^{(A) \operatorname{tr}}$, and the nonzero components $k_{I J K L}^{\mathrm{tr}}$ of the rank-four tensor $\boldsymbol{k}_{n+1}^{\mathrm{tr}}$ relative to the basis $\left\{\boldsymbol{n}_{n+1}^{(A) \text { tr }}\right\}$ are given by, $I, J, K, L=1,2,3$,

$$
\begin{aligned}
& k_{I I I}^{\mathrm{tr}}=-2 \tau_{I n+1}, \\
& k_{I J I J}^{\mathrm{tr}}=k_{I J J I}^{\mathrm{tr}}=\frac{\tau_{I n+1}\left(\lambda_{J n+1}^{\mathrm{etr}}\right)^{2}-\tau_{J n+1}\left(\lambda_{I n+1}^{\mathrm{etr}}\right)^{2}}{\left(\lambda_{I n+1}^{\mathrm{etr}}\right)^{2}-\left(\lambda_{J n+1}^{\mathrm{etr}}\right)^{2}} \quad \text { for } I \neq J,
\end{aligned}
$$

and where $a_{A B n+1}^{\mathrm{g}}$ are the components of the $3 \times 3$ matrix $\boldsymbol{a}_{n+1}^{\mathrm{g}}$ obtained by linearizing the local algorithm (29)

$$
\boldsymbol{a}_{n+1}^{\mathrm{g}}=\frac{\partial \hat{\tau}_{n+1}}{\partial \varepsilon_{n+1}^{\mathrm{etr}}} .
$$

This latter matrix is also model dependent, and it will also be detailed for each growth model example in the next section.

\section{REPRESENTATIVE NUMERICAL APPLICATIONS}

We apply in this section the preceding developments to two simple model examples of growth: a stress-dependent growth model, and a stress-free growth model. For both models, we choose the same hyperelastic behaviour for the soft tissue as given by the Hencky's model whose free energy is written as

$$
\psi\left(\boldsymbol{b}^{\mathrm{e}}\right)=\frac{1}{2} \kappa\left(\ln \left[J^{\mathrm{e}}\right]\right)^{2}+\mu \sum_{A=1}^{3}\left(\ln \left[\widetilde{\lambda}_{A}^{\mathrm{e}}\right]\right)^{2},
$$

where $\widetilde{\lambda}_{A}^{\mathrm{e}}=J^{\mathrm{e}^{-1 / 3}} \lambda_{A}^{\mathrm{e}}, A=1,2,3$, are the principal deviatoric elastic stretches, and $\kappa>0$ and $\mu>0$ are the bulk and shear moduli, respectively. After noticing that $\ln \left[J^{\mathrm{e}}\right]=\varepsilon_{1}^{\mathrm{e}}+\varepsilon_{2}^{\mathrm{e}}+\varepsilon_{3}^{\mathrm{e}}$, the elastic stress-strain relation in principal axes as given by (30) takes the following simple form

$$
\hat{\tau}=h \varepsilon^{\mathrm{e}}
$$

where the second order tensor $\boldsymbol{h}$ is given as

$$
\boldsymbol{h}=\kappa \mathbf{1} \otimes \mathbf{1}+2 \mu\left[\boldsymbol{I}-\frac{1}{3} \mathbf{1} \otimes \mathbf{1}\right]
$$


and where, and in all what follows, we have introduced the vector notation $\mathbf{1}=[1,1,1]^{T}$. Observe that $\boldsymbol{h}$ is constant for this particular hyperlastic behaviour.

\subsection{A stress-dependent growth model}

As a first modelling example, we consider a growth law inspired by the three equilibrium state model proposed in (1). Here, and within the present tensorial formulation, only one equilibrium state is considered for simplicity, say, the equilibrium around the normal physiological state of stress. The isotropic scalar valued function $g(\boldsymbol{\tau} ; \ldots)$ in eqn. (24) is chosen as

$$
g(\boldsymbol{\tau} ; J)=\eta\left\{\operatorname{tr}\left[\frac{\boldsymbol{\tau}}{J}\right]-p\right\} \equiv \eta\{\operatorname{tr}[\boldsymbol{\sigma}]-p\},
$$

where the parameter $p$ is the equilibrum (hydrostatic) stress state, $\eta$ is a viscous-like parameter which influences the rate of growth, and the dimension of which is $[\text { Pa.time }]^{-1}$. Hence, recalling the stress relation $\tau=J \sigma$, the growth is here governed by the true Cauchy stress state defined in the actual (deformed) configuration.

5.1.1. Algorithmic aspects. For this simple growth law, the logarithmic stretches update at the integration points level is explicit (step 3 of Table I)

$$
\varepsilon_{n+1}^{\mathrm{e}}=\left[\boldsymbol{I}+\delta_{n+1} \mathbf{1} \otimes \mathbf{1} \boldsymbol{h}\right]^{-1}\left\{\boldsymbol{\varepsilon}_{n+1}^{\mathrm{etr}}+\Delta t \eta p \mathbf{1}\right\},
$$

where we have used the notation $\delta_{n+1}=\eta \Delta t / J_{n+1}, \boldsymbol{h}$ is given by (37), and where $J_{n+1}=$ $\operatorname{det}\left[\boldsymbol{F}_{n+1}\right]$ is a known quantity at time $t_{n+1}$. Moreover, the algorithmic $3 \times 3$ matrix $\boldsymbol{a}_{n+1}^{\mathrm{g}}$ in step 5 of Table I is here given by

$$
\boldsymbol{a}_{n+1}^{\mathrm{g}}=\left[\boldsymbol{h}^{-1}+\delta_{n+1} \mathbf{1} \otimes \mathbf{1}\right]^{-1} \times\left[\boldsymbol{I}+\delta_{n+1}\left[\hat{\boldsymbol{\tau}}_{n+1} \cdot \mathbf{1}\right] \mathbf{1} \otimes \mathbf{1}\right],
$$

where one can notice that $\left[\hat{\boldsymbol{\tau}}_{n+1} \cdot \mathbf{1}\right]=\operatorname{tr}\left[\boldsymbol{\tau}_{n+1}\right]$. In deriving (40), use has been made of the kinematic relation $J_{n+1}=J_{n+1}^{\mathrm{e}} J_{n+1}^{\mathrm{g}}$ which, by algorithmic construction, is also equivalently written as $J_{n+1}=J_{n+1}^{\text {etr }} J_{n}^{\mathrm{g}}$. Hence, one obtains the differential identity $\mathrm{d} J_{n+1}=J_{n+1} 1 . \mathrm{d} \varepsilon_{n+1}^{\mathrm{etr}}$ which is used during the computation (34) to get (40).

5.1.2. Homogeneous growth example. As an illustration, we consider a $(10 \times 10 \times 10) \mathrm{mm}^{3}$ specimen discretized with $4 \times 4 \times 4$ common 8 -nodes brick elements. This sample is submitted to a uniform traction along the z-axis with the two other dimensions free (see Figure 2). For the soft tissue, we assume elastic properties of the same order as those employed for the modelling of arterial walls, see for example (27): $\kappa=2.5 \mathrm{MPa}$ and $\mu=1.15 \mathrm{MPa}$, i.e. with a Poisson's ration $\nu=0.375$. The equilibrium stress is chosen as $p=0.45 \mathrm{MPa}$.

Two series of computations are performed, the first one with a uniform prescribed nominal traction of value $0.6 \mathrm{MPa}$ (resultant $\overline{\mathrm{F}}=60 \mathrm{~N}$ ), and the second one with a prescribed nominal traction of value $0.2 \mathrm{MPa}$ (resultant $\bar{F}=20 \mathrm{~N}$ ). While the former loading exhibits growth, the later produces resorption, compare the loadings with the value of the normal physiological stress $p$. Figure 2 shows the results of the evolution of the Cauchy stress $\sigma_{33}$ for different values 
of the material viscous-like parameter $\eta$. In each computation, the stress decreases or increases asymptotically reaching the equilibrium value $p$.

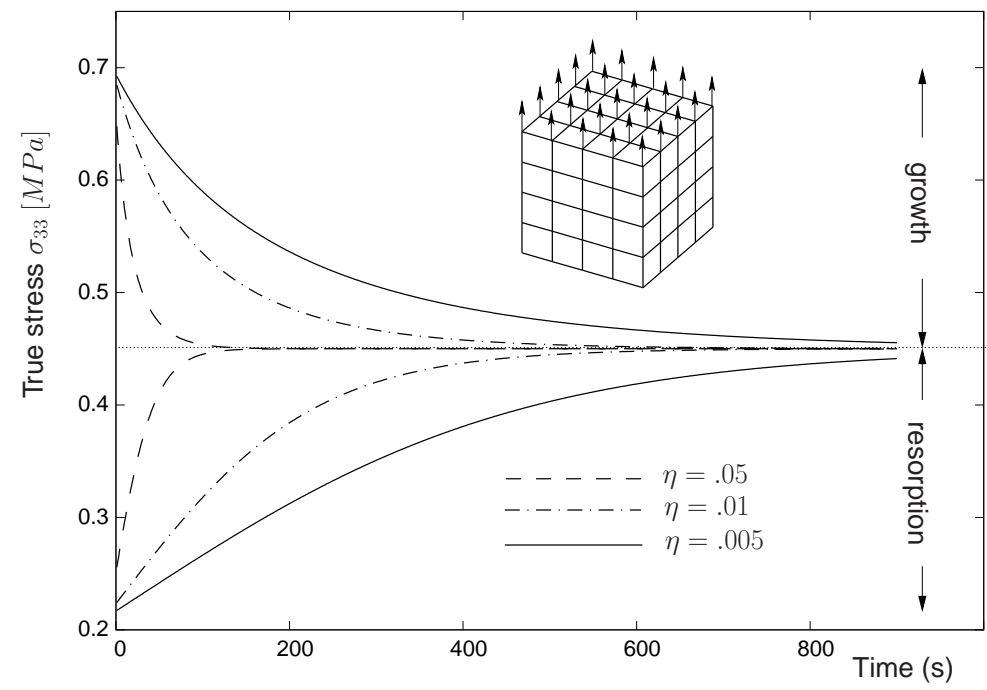

Figure 2. Evolution of the axial stress $\sigma_{33}$ in cases of growth or resorption for different values of the parameter $\eta\left[(M P a . s)^{-1}\right]$.

For instance, and as a geometrical illustration, Figures 3 and 4 show the evolutions of the deformations for growth and resorption, respectively, obtained with the particular value $\eta=$ $0.005(M P a . s)^{-1}$. Here again, one can observe that the tissue grows or resorbs until equilibrium is reached.



Figure 3. Evolution of the total volume $J$ and the axial streches $\left(\lambda_{1}=\lambda_{2}, \lambda_{3}\right)$ in the case of growth $(\bar{F}=60 N)$ with $\eta=0.005(M P a . s)^{-1}$.

5.1.3. A nonhomogeneous growth in a tube. As a second illustrative example, we consider the response of a long circular tube submitted to a prescribed constant internal pressure $\bar{p}_{i}$ in a selected 


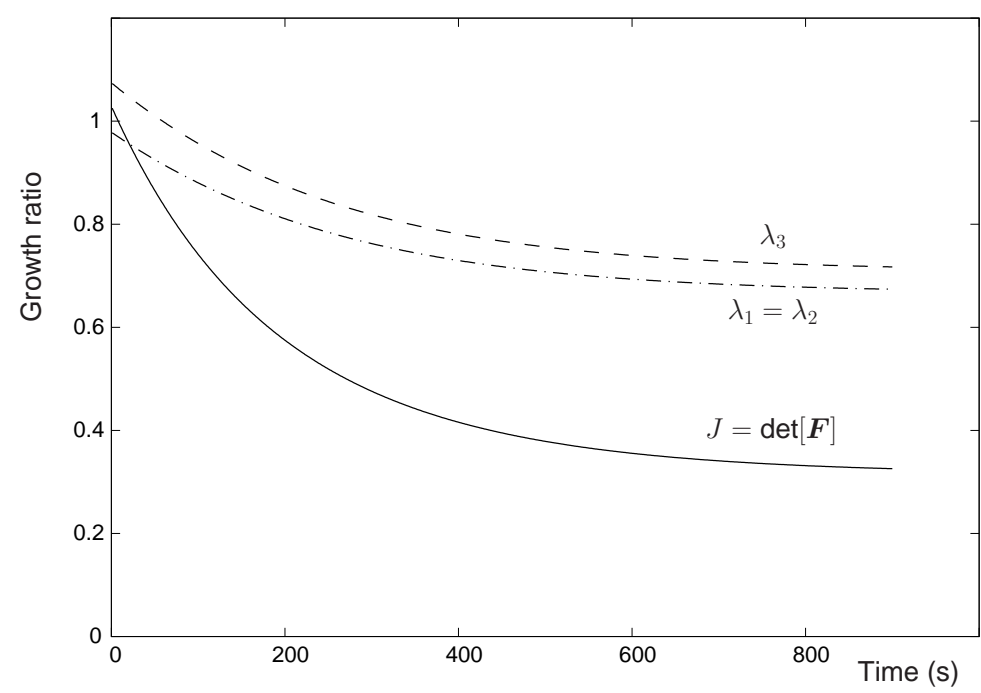

Figure 4. Evolution of the total volume $J$ and the axial streches $\left(\lambda_{1}=\lambda_{2}, \lambda_{3}\right)$ in the case of resorption $(\bar{F}=20 N)$ with $\eta=0.005(M P a . s)^{-1}$.

subsection in the middle. In the reference configuration, the internal radius is $R_{i}=4 \mathrm{~mm}$ and the thickness is $e=0.5 \mathrm{~mm}$. The lenght of the portion we consider in the computation is initially $H=30 \mathrm{~mm}$. For symmetry reasons, we choose to discretize one quarter of the tube with $8 \times 24$ common 8-nodes brick elements and three elements through the thickness, i.e. a total of 576 elements (see Figure 5).<smiles>CC(C)CC(C)C</smiles>
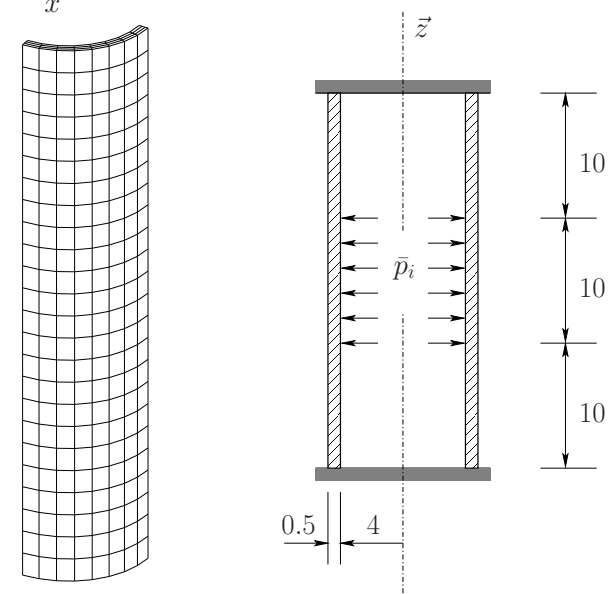

Figure 5. Circular tube submitted to an internal pressure. Geometry, finite element discretization, loading and boundary conditions.

For the hyperelastic behaviour of the tissue, use is made of the following parameters (see for example $(28 ; 29)$ for similar values adopted in the modelling of aretries): $\kappa=8.5 \mathrm{kPa}$ and $\mu=3.2 \mathrm{kPa}$, i.e. $\nu \approx 0.4$. And for the growth part, we choose the equilibrium stress $p=0.65 \mathrm{kPa}$ 
and the evolution parameter $\eta=510^{-4}(k P a . s)^{-1}$. Figure 6 depicts selective results obtained for the prescribed constant internal pressure of value $\bar{p}_{i}=0.2 \mathrm{kPa}$. The time increment was fixed to $\Delta t=1 \mathrm{~s}$ during the whole computation. One can observe a pronounced growth at areas of increased tension in the middle of the tube $\left(J^{\mathrm{g}}>1\right)$, and resorption at areas where the stress state were under the equilibrium stress at the initial state (the zones where $J^{\mathrm{g}}<1$ ).

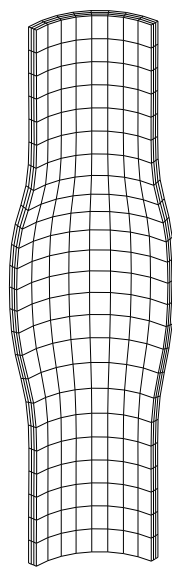

$10 \Delta t$

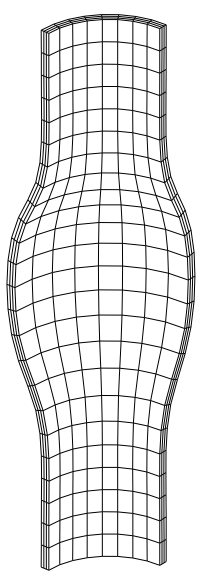

$200 \Delta t$

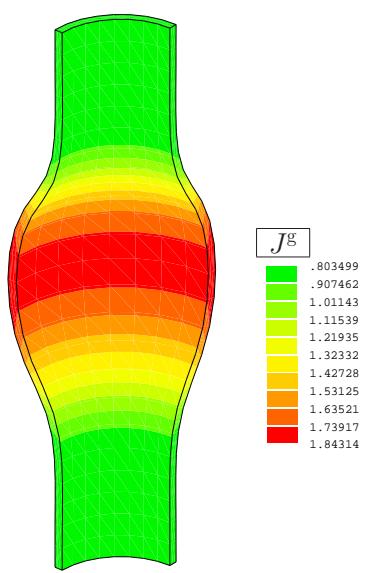

$400 \Delta t$

Figure 6. Qualitative simulation of growth. Different deformed meshes and growth field $J^{\mathrm{g}}=\operatorname{det}\left[\boldsymbol{F}^{\mathrm{g}}\right]$ at the last time step.

\subsection{A stress-independent growth model}

As a second modelling example, let us consider and discuss a growth law that is independent on the stress state. For instance, such a model could be interesting in describing growth in stress-free tissue culture, or in the normal developement of tissues from young to mature organisms. We consider the general scalar function $g(\tau ; \ldots)$ in eqn. (24) given as

$$
g\left(J^{\mathrm{g}}\right)=\eta\left(\delta-J^{\mathrm{g}}\right)^{\gamma},
$$

where the dimensionless $\delta$ is a growth equilibrium parameter, $\eta$ is again a viscous parameter influencing the rate of growth, the dimension of which is here [time $]^{-1}$, and the power $\gamma>0$ is a convenient dimensionless parameter. Hence, and as a first observation in this modelling design, the growth process stops when the growth volume $J^{\mathrm{g}}$ reaches the value $\delta$.

5.2.1. Algorithmic aspects. From the algorithmic point of view, the logarithmic stretches update (step 3 of Table I) is rewritten here as

$$
\boldsymbol{\varepsilon}_{n+1}^{\mathrm{e}}=\varepsilon_{n+1}^{\mathrm{etr}}-\Delta t \eta\left(\delta-\frac{J_{n+1}}{\exp \left[\varepsilon_{n+1}^{\mathrm{e}} \cdot \mathbf{1}\right]}\right)^{\gamma} \mathbf{1}
$$

where we have used the property $J_{n+1}^{\mathrm{e}}=\exp \left[\varepsilon_{n+1}^{\mathrm{e}} \cdot \mathbf{1}\right]$ together with the kinematic relation $J_{n+1}^{\mathrm{g}}=$ $J_{n+1} / J_{n+1}^{\mathrm{e}}$. This nonlinear equation can be solved locally by means of a Newton iterative procedure as summarized in Table II. The algorithmic $3 \times 3$ matrix $\boldsymbol{a}_{n+1}^{\mathrm{g}}$ (step 5 of Table I) takes here a 
Table II. Local logarithmic stretche update.

Given $J_{n+1}=\operatorname{det}\left[\boldsymbol{F}_{n+1}\right]$ and the time increment $\Delta t$, then

1. Initialize: $k=0, \boldsymbol{\varepsilon}_{n+1}^{\mathrm{e}(0)}=\boldsymbol{\varepsilon}_{n+1}^{\mathrm{etr}}$

2. Evaluate residual and check convergence

$$
\begin{aligned}
& \boldsymbol{r}_{n+1}^{(k)}=-\varepsilon_{n+1}^{\mathrm{e}(k)}+\varepsilon_{n+1}^{\mathrm{etr}}-\Delta t \eta\left(\delta-\frac{J_{n+1}}{\exp \left[\varepsilon_{n+1}^{\mathrm{e}(k)} \cdot \mathbf{1}\right]}\right)^{\gamma} \mathbf{1} \\
& \text { IF }\left\|\boldsymbol{r}_{n+1}^{(k)}\right\|>\text { TOL THEN go to Step } \mathbf{3} \\
& \text { ELSE set } \boldsymbol{\varepsilon}_{n+1}^{\mathrm{e}}=\boldsymbol{\varepsilon}_{n+1}^{\mathrm{e}(k)} \text { and EXIT. }
\end{aligned}
$$

3. Compute the tangent moduli and the increment

$$
\begin{aligned}
& \operatorname{set} \alpha_{n+1}^{(k)}=\frac{J_{n+1}}{\exp \left[\varepsilon_{n+1}^{\mathrm{e}(k)} \cdot \mathbf{1}\right]} \\
& \boldsymbol{D}_{n+1}^{(k)}=\left[\boldsymbol{I}+\Delta t \eta \gamma \alpha_{n+1}^{(k)}\left(\delta-\alpha_{n+1}^{(k)}\right)^{\gamma-1} \mathbf{1} \otimes \mathbf{1}\right]^{-1} \\
& \Delta \boldsymbol{\varepsilon}_{n+1}^{\mathrm{e}(k)}=\boldsymbol{D}_{n+1}^{(k)} \boldsymbol{r}_{n+1}^{(k)}
\end{aligned}
$$

4. Update the logarithmic elastic streches

$\varepsilon_{n+1}^{\mathrm{e}(k+1)}=\varepsilon_{n+1}^{\mathrm{e}(k)}+\Delta \varepsilon_{n+1}^{\mathrm{e}(k)}$

Set $k \longleftarrow k+1$ and return to Step 2 .

remarkably simple form

$$
\boldsymbol{a}_{n+1}^{\mathrm{g}}=\boldsymbol{h} .
$$

5.2.2. Uniform growth in a confined cylinder. We consider a plain cylinder confined in a rigid tube so that no radial expansion can occur. At the initial state, the cylinder matches perfectly the hollow tube with free initial stresses. Also, at time $t=0$, we consider that $\boldsymbol{F}_{0}^{\mathrm{g}}=\boldsymbol{I}$ so that $J_{0}^{\mathrm{g}}=1$. Then, as the tissue grows, the confinment generates volumic stresses until growth equilibrium is reached, i.e. when $J^{\mathrm{g}}=\delta$. This uniform analysis is conducted in two dimensions under the plane strain assumption, see Figure 7 for an illustration.

We use the following parameters for the hyperelastic behaviour of the tissue: $\kappa=6.14 \mathrm{MPa}$ and $\mu=2.3 \mathrm{MPa}$. And for the growth part, we choose the equilibrium growth to $\delta=2$ and fix the viscous parameter to $\eta=0.001 \mathrm{~s}^{-1}$, this later having the same role as in the precedent model. Figure 8 (top panel) shows the evolution of the growth volume $J^{\mathrm{g}}$ for different values of the exponent parameter $\gamma$. The more $\gamma$ is higher the more the growth process is slower. Hence, $\gamma$ can have the same influlence on the behaviour as the viscous parameter $\eta$, but the former is dimensionless as opposite to the later one. In each computation, $J^{\mathrm{g}}$ increases asymptotically until it reaches the model dependent given value $J^{\mathrm{g}}=\delta \equiv 2$. Also in Figure 8 (lower panel), one can observe that the corresponding internal pressure increases until an equilibrium stress is indireclty 




Figure 7. Confined growing cylinder. Plane strain two-dimensional analysis. Geometry and finite element mesh.

reached. This later is consequent of the structural effect of the problem as opposite to the normal physiological stress in the precedent growth law (in Section 5.1) which was an intrinsic material parameter. Finally, notice that due to the confinment, the Jacobian of the overall deformation $J=\operatorname{det}[\boldsymbol{F}]$ is equal to 1 during the whole growth process.

5.2.3. Residual stresses arising from growth. To gain more insight into the nature of the present stress-independent growth law, we study in this section the response of a growing free three-layer thick-walled tube. One can then expect that different growing behaviours between the layers will inevitably lead to resisual stresses within the tube thickness. To illustrate this fact qualitatively, we choose the geometry shown in Figure 9 and analyze its growth in two dimensions within the plane strain hypothesis.

The following table gives the material data used for each layer during the computation. The inner and outer layers have the same elasto-growth behaviour. Moreover, the three layers are chosen to have the same elastic behaviour. For the growth part, and for the sake of clarity, the exponent parameter $\gamma$ has been set to 1 for each layer. And for the other parameters, they have been chosen such that the inner and outer layers grow faster to their equilibrium growth than the intermediate one, on one hand, and to a different value of this equilibrium growth, on the other hand. The time increment was fixed to $\Delta t=1 \mathrm{~s}$ during the whole computation.

\begin{tabular}{|l|l|l|l|l|}
\hline Layer & $\kappa[M P a]$ & $\mu[M P a]$ & $\eta\left[s^{-1}\right]$ & $\delta$ \\
\hline inner \& outer & 6.14 & 2.3 & 0.01 & 1.5 \\
\hline intermediate & 6.14 & 2.3 & 0.001 & 3 \\
\hline
\end{tabular}

As an example of results, Figure 10(a) shows the distribution of the circumferential Cauchy stress through the three layers at an early stage where the growth of the inner and outer layers dominate, for instance here at $25 \Delta t$ where the deformed total thickness is $2.18 \mathrm{~mm}$. One can observe that this stress state is in compression in the the external layers, while it is in tension in the middle layer. This situation is reversed later on when growth in the outer and inner layers is in equilibrium while the middle layer continues growing untill a higher equilibrium value (here for $\delta=3$ ). Figure 10(b) shows this fact at $800 \Delta t$ where the total thickness is this time $2.86 \mathrm{~mm}$. 

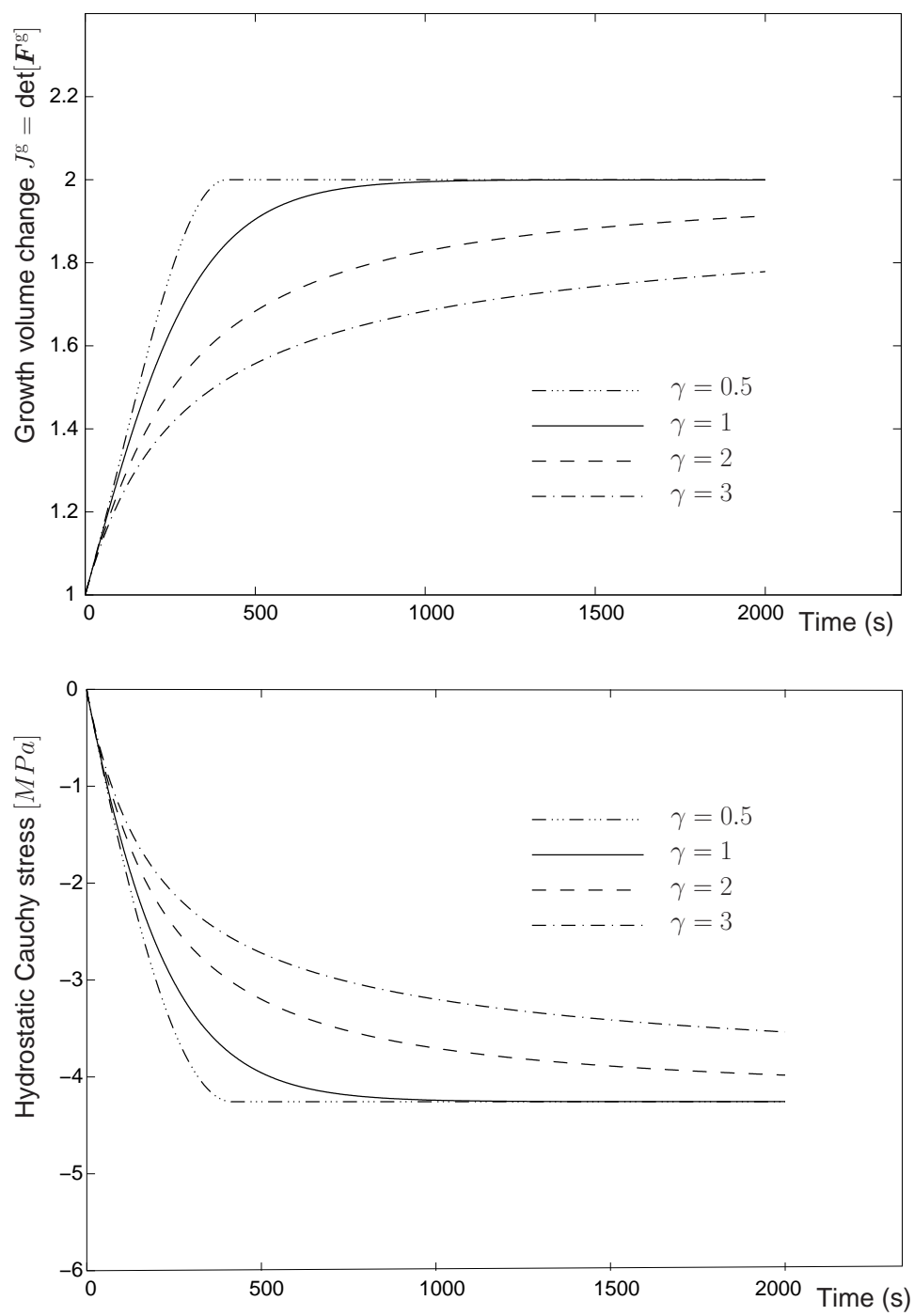

Figure 8. Evolution of the growth volume change $J^{\mathrm{g}}$ (top panel) and of the hydrostatic Cauchy stress (lower panel) for different values of the parameter $\gamma$.



Figure 9. Three-layer thick-walled tube. Geometrical data and finite element mesh. 


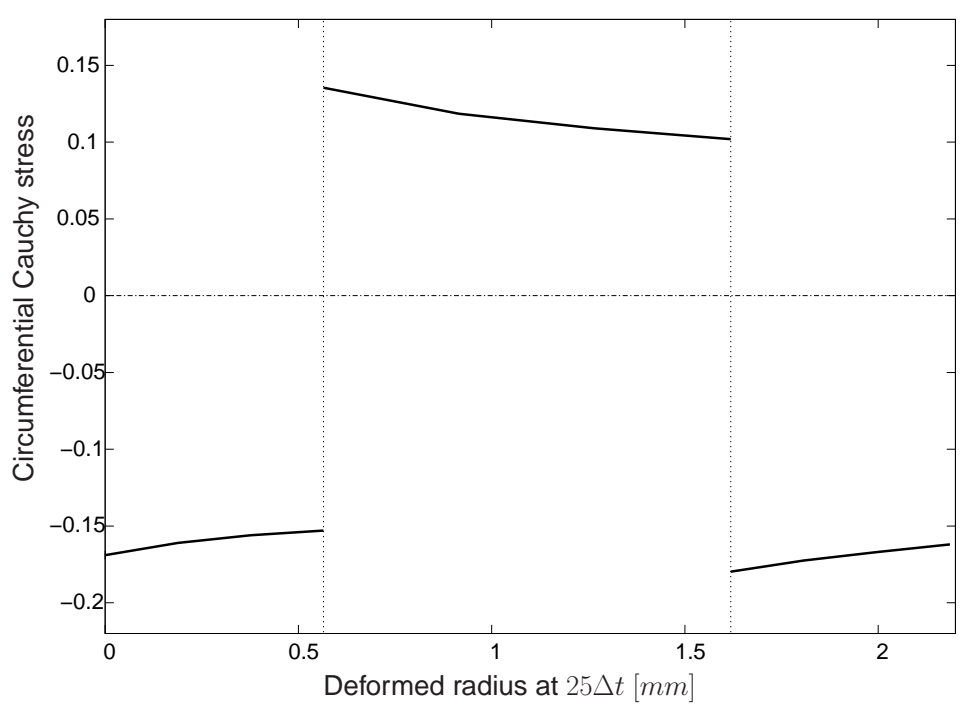

(a)

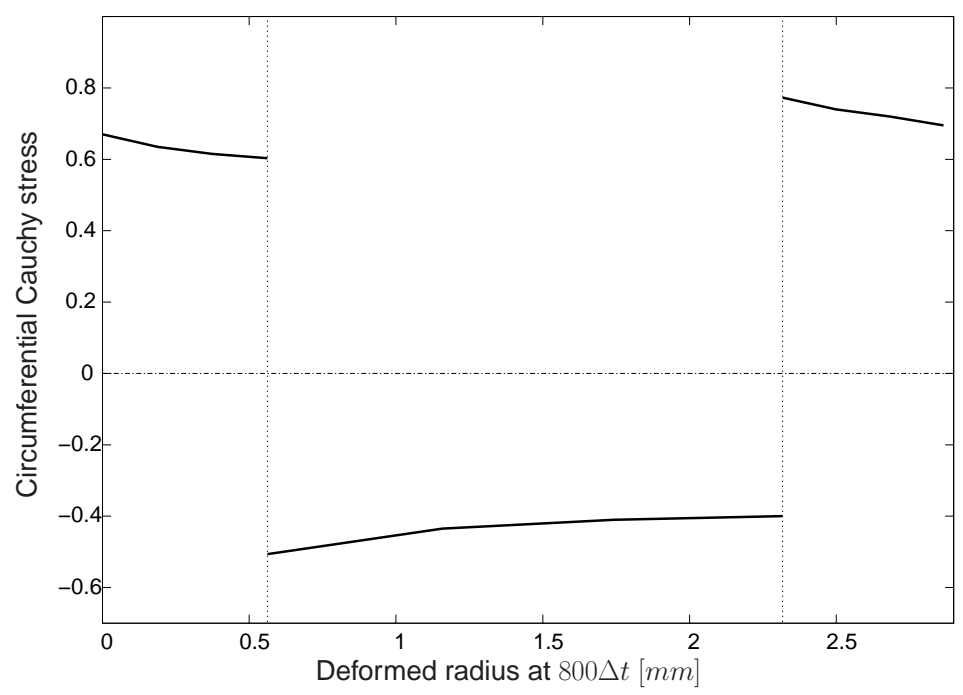

(b)

Figure 10. Plots of the residual stress vs deformed radius for the three-later thick-walled tube at $25 \Delta t$ (a), and $800 \Delta t(\mathrm{~b})$.

In all cases, this simple illustrative example shows that free growth of multi-layered soft tissues can exhibit residual stresses not only due to different elastic characteristics, but also because of the different growth behaviours within each layer.

\section{CONCLUDING REMARKS}

The main thrust of this paper has been the presentation of a thermodynamically sound formulation of growth designed for soft tissues at finite strains. Based on the nowadays well known kinematic notion of multiplicative decomposition of the deformation gradient into an elastic part and a growth 
part, standard arguments of continuum thermodynamics lead to a concise characterization of general growth evolution laws.

As an illustrative modelling example, the proposed methodology has been applied to model isotropic growth. Simple stress-dependent and stress-independent laws have been proposed and embeded into a finite element context. A set of representative numerical simulations has then been given to illustrate their effectiveness. Of course, and out of the scope of this paper, more complex growth laws can also be proposed within this framework.

Last but not least, the numerical effort is of the order of that of finite plastic computations. Moreover, the algorithms can simplify considerably when isotropy is considered. This has been shown through the detailed numerical algorithms given for the two growth laws used in this paper.

\section{REFERENCES}

[1] Fung Y. Biomechanics: motion, flow, stress and growth. Springer: Berlin Heidelberg New York, 1990.

[2] Taber L. Biomechanics of growth, remodeling, and morphogenesis. ASME Appl. Mech. Rev. 1995; 48(8):487-545.

[3] Cowin S. How is a tissue built? J. Biomech Eng. 2000; 122:553-569.

[4] Cowin S, Humphrey J. (eds.) Cardiovascular soft tissue mechanics. Kluwer: Dordrecht, 2000.

[5] Holzapfel G, Ogden R. (eds.) Biomechanics of soft tissue in cardiovascular systems. CISM courses and lectures number 441. Springer: Berlin Heidelberg New York, 2003.

[6] Rodriguez E, Hoger A, McCulloch A. Stress-dependent finite growth in soft elastic tissues. $J$. Biomechanics 1994; 27(4):455-467.

[7] Chen Y, Hoger A. Constitutive functions of elastic materials in finite growth and deformation. J. Elasticity 2000; 95:175-193.

[8] Klisch S, Dyke TV, Hoger A. A theory of volumetric growth for compressible elastic biological materials. Math. Mech. Solids 2001; 6:551-575.

[9] Himpel G, Kuhl E, Menzel A, Steinmann P. Computational modelling of isotropic multiplicative growth. Comput. Model. Eng. Sci. 2005; 8(2):119-134.

[10] Hsu F. The influences of mechanical loads on the form of a growing elastic body. $J$. Biomechanics 1968; 1:303-311.

[11] Cowin S, Firoozbakhsh K. Bone remodeling of diaphyseal surfaces under constant load: theoretical predictions. J. Biomechanics 1981; 7:471-484.

[12] Taber L, Eggers D. Theoretical study of stress-modulated growth in the aorta. J. Theo. Biol. 1996; 180:343-357. 
[13] Rachev A, Stergiopulos N, Meister J. Theoretical study of dynamics of arterial wall remodeling in response to change of blood pressure. J. Biomechanics 1996; 29(5):635-642.

[14] Truesdell C, Noll W. The nonlinear field theories of mechanics. Handbuch der Physik Bd. III/3, Fluegge S (ed.), Springer-Verlag, Berlin, 1965.

[15] Karšaj I, Sansour C, Soric̀ J. The modelling of fibre reorientation in soft tissue. Biomech. Model Mechanobiol. 2009; 8(5):359-370.

[16] Sansour C, Karšaj I, Soric̀ J. A formulation of anisotropic continuum elastoplasticity at finite strains. Part I: Modelling. Int. J. of Plasticity 2006; 22:2346-2365.

[17] Coleman B, Gurtin M. Thermodynamics with internal variables. Journal of Chemics and Physics 1967; 47:597-613.

[18] Germain P, Nguyen Q, Suquet P. Continuum thermodynamics. ASME Journal of Applied Mechanics 1983; 50:1010-1021.

[19] Sansour C, Kollmann F. Large viscoplastic deformation of shells. Theory and finite element formulation. Computational Mechanics 1998; 21:512-525.

[20] Sansour C, Wagner W. A model for finite strain viscoplasticity based on unified constitutive equations. Theoretical and computational considerations with application to shells. Comput. Methods Appl. Mech. Engrg. 2001; 191:423-450.

[21] Chadwick P. Continuum mechanics. Concise theory and problems. John Wiley and Sons, Inc. (Dover Publications, Inc.): New York (New York), 1976 (1999).

[22] Marsden J, Hughes T. Mathematical foundations of elasticity. Prentice-Hall, EnglewoodCliffs: New Jersey, 1983.

[23] Skalak R, Dasgupta G, Moss M, Otten E, Dullemeijer P, Vilmann H. Analytical description of growth. J. Theor. Biol. 1982; 94:555-577.

[24] Simo J. Numerical analysis and simulation of plasticity. Handbook of Numerical Analysis, vol. VI, Ciarlet P, Lions J (eds.), North-Holland, 1998; 183-499.

[25] Armero F. Elastoplastic and viscoplastic deformations in solids and structures. Encyclopedia of computational mechanics, vol. 2: Solids and Structures, Stein E, de Borst R, Hughes T (eds.), John Wiley \& Sons., 2004; 227-266.

[26] Nedjar B. Frameworks for finite strain viscoelastic-plasticity based on multiplicative decompositions. Part II: Computational aspects. Comput. Methods Appl. Mech. Eng. 2002; 191:1563-1593.

[27] Kuhl E, Maas R, Himpel G, Menzel A. Computational modeling of arterial wall growth. Biomech. Model Mechanobiol. 2007; 6(5):321-331.

[28] Holzapfel G. Computational biomechanics of soft biological tissue. Encyclopedia of computational mechanics, vol. 2: Solids and Structures, Stein E, de Borst R, Hughes T (eds.), John Wiley \& Sons., 2004; 605-635. 
[29] Menzel A. A fibre reorientation model for orthotropic multiplicative growth. Biomech. Model Mechanobiol. 2007; 6(5):303-320. 